\title{
Seminare/Séminaires/Seminari 2018
}

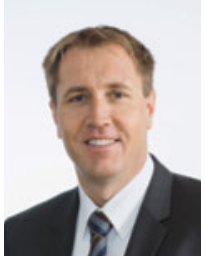

Bild: René Häller,

Geschäftsführer FMH Consulting Services AG

\section{Praxiseröffnung/-übernahme}

Zielgruppe: Ärztinnen und Ärzte, die vor einer Praxiseröffnung (Einzel-/Gruppenpraxis), dem Einstieg in eine Gruppenpraxis oder vor einer Praxisübernahme stehen.

\section{Themen}

- Juristische Aspekte (Bewilligungen, Zulassungen, Verträge)

- Gesellschaftsformen / Ehe- und Erbrecht (Privat-/Geschäftsvermögen, Güterstand, Erbschaftsplanung)

- Praxiseinrichtung (Inneneinrichtung, Kosten)

- Praxisadministration (Leistungserfassungsund Abrechnungssysteme)

- Bewertung einer Arztpraxis als Verhandlungsbasis

- Finanzierung der Arztpraxis (Businessplan, Kredite, Absicherung)

- Versicherungen/Vorsorge/Vermögen (Personen- und Sachversicherungen, Vorsorgeplanung)

\begin{tabular}{|c|c|c|}
\hline Daten & & \\
\hline K01 & $\begin{array}{l}\text { Donnerstag, } \\
\text { 1. März } 2018 \\
09.00-16.30 \mathrm{Uhr}\end{array}$ & $\begin{array}{l}\text { Zürich } \\
\text { Volkshaus }\end{array}$ \\
\hline К02 & $\begin{array}{l}\text { Donnerstag, } \\
\text { 17. Mai } 2018 \\
\text { 16.00-20.30 Uhr }\end{array}$ & $\begin{array}{l}\text { St.Gallen } \\
\text { Hotel Einstein }\end{array}$ \\
\hline К03 & $\begin{array}{l}\text { Donnerstag, } \\
\text { 7. Juni } 2018 \\
09.00-16.30 \text { Uhr }\end{array}$ & $\begin{array}{l}\text { Bern } \\
\text { Schmiedstube }\end{array}$ \\
\hline K04 & $\begin{array}{l}\text { Donnerstag, } \\
\text { 6. September } 2018 \\
09.00-16.30 \text { Uhr }\end{array}$ & $\begin{array}{l}\text { Zürich } \\
\text { Volkshaus }\end{array}$ \\
\hline К05 & $\begin{array}{l}\text { Donnerstag, } \\
\text { 8. November } 2018 \\
09.00-16.30 \mathrm{Uhr}\end{array}$ & $\begin{array}{l}\text { Basel } \\
\text { Hotel Victoria }\end{array}$ \\
\hline
\end{tabular}

Praxisübergabe/-aufgabe

Zielgruppe: Ärztinnen und Ärzte, die ihre Praxis an einen Partner/Nachfolger übergeben oder liquidieren wollen. Idealtermin: 5-10 Jahre vor geplanter Übergabe oder allfälliger Liquidation (aus steuertechnischen und vorsorgeplanerischen Gründen).

Wären Sie gerne Teil einer Gruppenpraxis - sei dies als Mitarbeitender oder als Partner? Oder ziehen Sie vor, eine bestehende Praxis zu übernehmen oder Ihre eigene Praxis ganz neu aufzubauen? Möchten Sie die Zukunft Ihrer bestehenden Praxis vorzeitig sichern und/oder die Praxisübergabe bzw. die Schliessung Ihrer Praxis steuertechnisch optimal vorbereiten? Unsere grossenteils kostenlosen Seminare helfen Ihnen, diese und andere Fragen rund um die Gründung/Übernahme bzw. die Übergabe/ Aufgabe zu beantworten.

Souhaitez-vous rejoindre un cabinet de groupe? Comme collaborateur ou comme partenaire? Ou préféreriez-vous reprendre un cabinet ou ouvrir le vôtre? Souhaitez-vous assurer l'avenir de votre cabinet médical et/ou optimiser fiscalement sa remise ou sa fermeture? Nos séminaires, dont une grande partie est gratuite, vous aideront à répondre à ces questions et à d'autres sur l'ouverture / la reprise ou la remise / la fermeture de cabinets médicaux.

\section{Themen}

- Praxispartner- oder Nachfolgesuche (projektorientiertes Vorgehen)

- Juristische Aspekte (Verträge, Krankengeschichten)

- Bewertung einer Arztpraxis als Verhandlungsbasis

- Versicherungen/Vorsorge/Vermögen (Übergabe/Auflösung von Versicherungsverträgen, Pensions- und Finanzplanung)

- Steuern (Steueroptimierung bei der Praxisübergabe oder Liquidation)

\begin{tabular}{|c|c|c|}
\hline Daten & & \\
\hline K06 & $\begin{array}{l}\text { Donnerstag, } \\
\text { 8. März } 2018 \\
\text { 13.30-18.00 Uhr }\end{array}$ & $\begin{array}{l}\text { Zürich } \\
\text { Volkshaus }\end{array}$ \\
\hline K07 & $\begin{array}{l}\text { Donnerstag, } \\
\text { 3. Mai } 2018 \\
16.00-20.30 \text { Uhr }\end{array}$ & $\begin{array}{l}\text { St. Gallen } \\
\text { Hotel Einstein }\end{array}$ \\
\hline К08 & $\begin{array}{l}\text { Donnerstag, } \\
\text { 14. Juni } 2018 \\
\text { 13.30-18.00 Uhr }\end{array}$ & $\begin{array}{l}\text { Bern } \\
\text { Schmiedstube }\end{array}$ \\
\hline К09 & $\begin{array}{l}\text { Donnerstag, } \\
\text { 13. September } 2018 \\
\text { 13.30-18.00 Uhr }\end{array}$ & $\begin{array}{l}\text { Zürich } \\
\text { Volkshaus }\end{array}$ \\
\hline K10 & $\begin{array}{l}\text { Donnerstag, } \\
\text { 22. November } 2018 \\
\text { 13.30-18.00 Uhr }\end{array}$ & $\begin{array}{l}\text { Basel } \\
\text { Hotel Victoria }\end{array}$ \\
\hline
\end{tabular}

\section{Gruppenpraxis}

Zielgruppe: In Ausbildung stehende Ärztinnen und Ärzte, die sich einer Gruppenpraxis anschliessen wollen, sowie praxistätige Ärztinnen und Ärzte, die ihre Einzelpraxis an eine Gruppenpraxis anschliessen wollen.

\section{Themen}

- Strategie (Ziele und Angebot)

- Unternehmer (Team, Verhaltensregeln; finanzielle Beteiligung und Entschädigung)

- Finanzen und Recht (Versicherung, Vorsorge und Vermögen; Rechtsform, Finanzen, Steuern)

- Standort, Praxisobjekt (Anforderungen, Konkurrenzanalyse; Einrichtung, Kosten)

- Personal (Qualifikationen; Gesetze, Reglemente, Verträge)
- Führung und Organisation (Struktur und Abläufe; Aufgaben, Verantwortungen, Kompetenzen)

- EDV und Administration (Praxisinformatik: Anforderungen und Evaluation)

- Praxisbericht (Erfahrungsbericht eines Arztes, Mitgründer einer Gruppenpraxis aus der Region)

\begin{tabular}{lll}
\hline Daten & & \\
\hline K80 & Donnerstag, & Luzern \\
& 24. Mai 2018 & Hotel \\
& 13.30-18.00 Uhr & Continental-Park \\
K81 & Donnerstag, & Aarau \\
& 22. November 2018 & Gasthof \\
& 13.30-18.00 Uhr & zum Schützen
\end{tabular}

Praxiscomputer-Workshop

Zielgruppe: Ärztinnen und Ärzte, die vor einer Praxiseröffnung/-übernahme stehen oder bereits praxistätig sind.

Themen

- Anforderungen an ein Praxisinformationssystem

- Evaluationsprozess (projektorientiertes Vorgehen)

- Präsentation von sieben führenden Praxisinformationssystemen (Leistungserfassung, Abrechnen, Agenda, Statistik, Geräteeinbindung, Krankengeschichte, Finanzbuchhaltung usw.)

\begin{tabular}{lll}
\hline Daten & & \\
\hline K13 & $\begin{array}{l}\text { Donnerstag, } \\
\text { 22. März 2018 }\end{array}$ & Zürich \\
& $\begin{array}{l}\text { 13.15-17.45 Uhr } \\
\text { K14 }\end{array}$ & Technopark \\
& $\begin{array}{l}\text { Donnerstag, } \\
\text { 28. Juni 2018 }\end{array}$ & \\
& 13.15-17.45 Uhr & Bern \\
K15 & Donnerstag, & \\
& 15. November 2018 & Olten \\
& 13.15-17.45 Uhr & Stadttheater
\end{tabular}

Going paperless - Digitalisierung in der Praxis

Zielgruppe: Ärztinnen und Ärzte, die ihre Praxis mit digitalen Krankengeschichten führen wollen. 
Themen

- Struktur und Organisation (Strategische Ausrichtung, Vor- und Nachteile der Digitalisierung, Geschäftsprozesse und Informationsfluss)

- Erstellen eines Anforderungskataloges (Lastenheft) und seine Verwendungszwecke

- Das Führen der elektronischen Krankengeschichte (Aufbau und Struktur)

Kosten: 500 CHF (inkl. Unterlagen und Verpflegung).

\begin{tabular}{lll}
\hline Daten & & \\
\hline K92 & Mittwoch, & \\
& 14. März 2018 & Zürich \\
& 09.00-16.30 Uhr & Volkshaus \\
K93 & $\begin{array}{l}\text { Mittwoch, } \\
\text { 5. September 2018 }\end{array}$ & Bern \\
& 09.00-16.30 Uhr & Schmiedstube \\
\end{tabular}

\section{Röntgen in der Arztpraxis}

Zielgruppe: Ärztinnen und Ärzte, die vor einer Praxiseröffnung/-übernahme stehen oder bereits praxistätig sind.

\section{Themen}

- Rentabilität Röntgen in der Arztpraxis

- Evaluation und Beschaffung neuer oder gebrauchter Anlagen

- Möglichkeiten der Umrüstung von analogen zu digitalen Anlagen

- Vor- und Nachteile analoger und digitaler Systeme

- Komplette Marktübersicht mit Preisen und Leistungskomponenten

\begin{tabular}{lll}
\hline Datum & & \\
\hline K16 & Donnerstag, & \\
& 30. August 2018 & Niederscherli \\
& 09.30-16.00 Uhr & digitalXray AG
\end{tabular}

Tarifwerk TARMED - Einführungskurs

Zielgruppe: Ärztinnen und Ärzte mit bestehender Praxis und solche, die kurz vor einer Praxiseröffnung oder Praxisübernahme stehen. Der Einführungskurs vermittelt den Kursteilnehmern die Grundlagen des Tarifwerkes TARMED.

\section{Themen}

- Fakten (gesetzliche und vertragliche Grundlagen)

- Struktur (Tarifbrowser, Grundstruktur, Regelhierarchie, Leistungsblöcke, Leistungsgruppen)

- Generelle Interpretationen («Allgemeine Grundleistungen", "Hauptleistungen, Zuschlagsleistungen», "Nichtärztliche Leistungserbringung» usw.)

- Parameter einer Tarifposition ("Quantitative und Qualitative Dignität», "Ärztliche Leistung AL", «Assistenz», «Raumbelegung» usw.)

- Tarifpositionen aus dem Kapitel 00 Grundleistungen

- Praxislabor und Präsenzdiagnostik
- Organisationen und Informationsquellen Kosten: 200 CHF (inkl. Unterlagen).

\begin{tabular}{|c|c|c|}
\hline Daten & & \\
\hline K70 & $\begin{array}{l}\text { Dienstag, } \\
\text { 6. März } 2018 \\
\text { 13.30-16.45 Uhr }\end{array}$ & $\begin{array}{l}\text { Olten } \\
\text { Hotel Arte }\end{array}$ \\
\hline K71 & $\begin{array}{l}\text { Dienstag, } \\
\text { 8. Mai } 2018 \\
13.30-16.45 \text { Uhr }\end{array}$ & $\begin{array}{l}\text { Olten } \\
\text { Hotel Arte }\end{array}$ \\
\hline K72 & $\begin{array}{l}\text { Dienstag, } \\
\text { 4. September } 2018 \\
13.30-16.45 \text { Uhr }\end{array}$ & $\begin{array}{l}\text { Olten } \\
\text { Hotel Arte }\end{array}$ \\
\hline K73 & $\begin{array}{l}\text { Dienstag, } \\
\text { 23. Oktober } 2018 \\
\text { 13.30-16.45 Uhr }\end{array}$ & $\begin{array}{l}\text { Olten } \\
\text { Hotel Arte }\end{array}$ \\
\hline
\end{tabular}

\section{Marketing für Ärztinnen und Ärzte}

Zielgruppe: Ärztinnen und Ärzte, die vor einer Praxiseröffnung stehen oder bereits eine Praxis führen und diese unternehmerisch betreiben möchten.

\section{Themen}

- Verändertes Umfeld (Gesundheitsmarktsituation und Trends)

- Marketing heisst gesehen werden (Wofür steht meine Praxis? Wie definiere ich mich?)

- Einführung ins Branding (Je besser die Analyse, desto treffsicherer die strategische Positionierung)

- Juristische Hürden (Nach welchen rechtlichen Grundlagen richtet sich die ärztliche Werbung?)

- Kommunikationsplanung (In welchen Medien und auf welchen Werbeträgern sollen wir kommunizieren?)

- Marketing in der digitalen Welt (Welches sind die Chancen und Risiken?)

\begin{tabular}{lll}
\hline Daten & & \\
\hline K63 & Donnerstag, & \\
& 8. März 2018 & Zürich \\
& 13.30-17.00 Uhr & Lumina \\
K64 & Donnerstag, & Nottwil \\
& 22. November 2018 & Hotel \\
& 13.30-17.00 Uhr & Sempachersee \\
& & \\
\hline
\end{tabular}

Telefonseminar für MPA (bzw. Praxisteam)

Zielgruppe: MPA mit telefonischem Kundenkontakt sowie Auszubildende, die zum professionellen Telefonieren angeleitet werden sollen

\section{Themen}

- Die medizinische Praxisassistentin als Visitenkarte der Praxis

- Image der Arztpraxis. MPA repräsentieren die Unternehmenskultur, organisieren die Praxis und sind somit ein wesentlicher Bestandteil für den Unternehmenserfolg.

- Bedeutung des ersten Telefonkontakts

- Richtig telefonieren - eine Anleitung

Kosten: 150 CHF (inkl. Kursunterlagen und Verpflegung).

\begin{tabular}{lll}
\hline Daten & & \\
\hline K65 & Donnerstag, & \\
& 22. März 2018 & Bauma \\
& 09.00-16.30 Uhr & prama GmbH \\
K66 & Donnerstag, & Nottwil \\
& 15. November 2018 & Hotel \\
& $09.00-16.30$ Uhr & Sempachersee \\
\hline
\end{tabular}

Kommunikation mit Patienten für MPA (bzw. Praxisteam)

Zielgruppe: MPA sowie Auszubildende, die zu einer professionelleren Kommunikation mit Patienten (auch schwierigen) angeleitet werden sollen und sich in der Kommunikation im Team verbessern möchten.

\section{Themen}

- Kommunikative Techniken (aktives Zuhören, 4-Ohren-Prinzip)

- Kundenorientierte Kommunikation

- Eigenes Konfliktverhalten

- Positiver Umgang mit Reklamationen und schwierigen Gesprächssituationen

Kosten: 150 CHF (inkl. Kursunterlagen und Verpflegung)

\begin{tabular}{lll}
\hline Daten & & \\
\hline K67 & Freitag, & \\
& 9. März 2018 & Bauma \\
& 09.00-16.30 Uhr & prama GmbH \\
K68 & Donnerstag, & Nottwil \\
& 6. September 2018 & Hotel \\
& 09.00-16.30 Uhr & Sempachersee
\end{tabular}

Kranken- und Sozialversicherungen für MPA (bzw. Praxisteam)

Zielgruppe: MPA und andere Praxismitarbeitende, die sich in der komplexen Materie besser zurechtfinden und Zusammenhänge schneller erkennen möchten.

Themen

- Gesundheitswesen Schweiz (Übersicht Kranken- u. Sozialversicherungen, Kosten u. Finanzierung)

- Versicherte Leistungen KVG / VVG (Voraussetzungen zur Leistungsübernahme (u.a. WZW), Unterschiede KVG / VVG und Leistungslücken)

- Merkmale und Abgrenzungen Unfall/ Krankheit (Unfallerkennung, Koordination mit anderen Sozialversicherungen)

Kosten: 300 CHF (inkl. Unterlagen und Verpflegung). Praxisteams erhalten einen Rabatt von $10 \%$ pro Teilnehmer/-in.

\begin{tabular}{lll}
\hline Daten & & \\
\hline K60 & $\begin{array}{l}\text { Donnerstag, } \\
\text { 14. Juni 2018 }\end{array}$ & Zürich \\
& $\begin{array}{l}\text { 09.00-16.30 Uhr } \\
\text { Donnerstag, }\end{array}$ & Volkshaus \\
K61 & 8. November 2018 & Luzern \\
& 09.00-16.30 Uhr & HFT Luzern AG
\end{tabular}


Ouverture et reprise d'un cabinet médical

Groupe cible: Médecins sur le point d'ouvrir un cabinet médical (individuel ou de groupe), de joindre un cabinet de groupe ou de reprendre un cabinet existant.

\section{Contenu}

- Aspects juridiques (contrats, autorisations, admission à pratiquer, dossier patients)

- Business plan (plan de financement, crédit d'exploitation, financement par la banque)

- Aménagement (implantation, projet et concept d'aménagement, mobilier, budget)

- Estimation d'un cabinet comme base de négociations

- Laboratoire

- Administration d'un cabinet médical

- Assurances

- Passage du statut de salarié à celui d'indépendant

- Fiscalité

\begin{tabular}{|c|c|c|}
\hline Dates & & \\
\hline \multirow[t]{3}{*}{ K20 } & Jeudi & Lausanne \\
\hline & $1^{\mathrm{er}} \operatorname{mars} 2018$ & World Trade \\
\hline & $13.30-18.30 \mathrm{~h}$ & Center \\
\hline \multirow[t]{3}{*}{ K21 } & Jeudi & \\
\hline & 7 juin 2018 & Genève \\
\hline & $13.30-18.30 \mathrm{~h}$ & Hôtel Warwick \\
\hline \multirow[t]{3}{*}{ K22 } & Jeudi & Lausanne \\
\hline & 13 septembre 2018 & World Trade \\
\hline & $13.30-18.30 \mathrm{~h}$ & Center \\
\hline \multirow[t]{3}{*}{ K23 } & Jeudi & \\
\hline & 8 novembre 2018 & Genève \\
\hline & $13.30-18.30 \mathrm{~h}$ & Crowne Plaza \\
\hline
\end{tabular}

Remise et cessation d'un cabinet médical

Groupe cible: Médecins désirant remettre un cabinet médical à un associé ou à un successeur ou qui doivent fermer leur cabinet médical. Idéalement 5-10 ans avant la remise/cessation prévue (pour des questions de taxation et prévoyance).

\section{Contenu}

- Recherche active d'un successeur/associé

- Aspects juridiques (contrats, dossiers médicaux)

- Estimation d'un cabinet comme base de négociations

- Assurances/prévoyance/patrimoine (remise/résiliation des contrats d'assurances, prévoyance, planification de la retraite et des finances)

- Conséquences fiscales d'une remise ou d'une cessation (optimisation de l'impact fiscale lors d'une remise/cessation)

\begin{tabular}{lll}
\hline Dates & & \\
\hline K24 & Jeudi & Lausanne \\
& 17 mai 2018 & World Trade \\
K25 & J.30-18.30 h & Center \\
& Jeudi & \\
& 15 novembre 2018 & Genève \\
& 13.30-18.30 h & Crowne Plaza \\
\hline
\end{tabular}

\section{Cabinet de groupe}

Groupe cible: Médecins en formation voulant exercer leur future activité en cabinet de groupe et libres praticiens qui souhaitent affilier leur cabinet individuel à un cabinet de groupe.

\section{Contenu}

- Stratégie (objectifs et prestations)

- Entrepreneurs (équipe, règles de conduite; participation financière et rémunération)

- Finances et droit (assurances, prévoyance et patrimoine; forme juridique, finances et impôts)

- Lieu d'implantation et immobilier (exigences; aménagement et coûts, laboratoire)

- Ressources humaines (qualifications; lois règlements et contrats)

- Direction et organisation (structure et processus; tâches, responsabilités et compétences)

- Informatique et administration (système informatique pour le cabinet: attentes et évaluation)

- Rapport d'expérience (rapport de l'expérience d'un médecin, co-fondateur d'un cabinet de groupe de la région)

\begin{tabular}{lll}
\hline Dates & & \\
\hline K85 & Jeudi & \\
& 26 avril 2018 & Genève \\
& 13.30-18.30 h & Hôtel Warwick \\
K86 & Jeudi & Lausanne \\
& 27 septembre 2018 & World Trade \\
& 13.30-18.30 h & Center
\end{tabular}

Atelier consacré à l'informatique au cabinet médical

Groupe cible: Médecins sur le point d'ouvrir un cabinet médical et médecins déjà établis qui veulent changer leur logiciel.

\section{Contenu}

- Evaluation d'un système de gestion de cabinet (introduction)

- Présentation de cinq logiciels pour la gestion du cabinet (gestion des données des patients, de la facturation et de l'encaissement, statistiques, gestion de l'agenda, connexion des appareils médicaux, etc.)

\begin{tabular}{lll}
\hline Date & & \\
\hline K28 & Jeudi & Lausanne \\
& 21 juin 2018 & World Trade \\
& $13.30-18.00 \mathrm{~h}$ & Center
\end{tabular}

Apertura e rilevamento di uno studio medico

Gruppo destinatario: Medici in procinto di aprire o di rilevare uno studio medico.

\section{Contenuto}

- Business plan (preparazione del piano di finanziamento e del credito d'esercizio, prestito bancario)

- Pianificazione (insediamento, progetto e pianificazione, mobilio, budget)

- Valutazione di uno studio medico

- Amministrazione di uno studio medico (interna allo studio, rapporti con la banca)

- Assicurazioni (assicurazioni necessarie interne ed esterne allo studio)

- Passaggio dallo stato di dipendente a quello di indipendente

- Fiscalità

\begin{tabular}{lll}
\hline Date & & \\
\hline K50 & Mercoledi & Chiasso \\
& $\begin{array}{l}\text { 18 aprile } 2018 \\
\text { dalle } 14.00 \text { alle 17.00 }\end{array}$ & $\begin{array}{l}\text { FMH Services } \\
\text { (Consulting) }\end{array}$ \\
K51 & $\begin{array}{l}\text { Mercoledi } \\
\text { 17 ottobre 2018 } \\
\text { dalle 14.00 alle 17.00 }\end{array}$ & $\begin{array}{l}\text { Chiasso } \\
\text { (CMH Services }\end{array}$ \\
& & \\
\hline
\end{tabular}

Studio medico di gruppo

Gruppo destinatario: Medici in formazione che desiderano esercitare la loro futura attività in uno studio medico associato. Liberi praticanti che auspicano affiliare o che hanno già affiliato il loro studio medico individuale ad uno associato.

\section{Contenuto}

- Strategia (obiettivi e prestazioni)

- Imprenditori (composizione del gruppo di lavoro e regole di condotta; partecipazione finanziaria e metodi di remunerazione)

- Finanze e diritto (assicurazioni, previdenza e patrimonio; forma giuridica, finanze e fiscalità)

- Ubicazione e allestimento dello studio (esigenze e analisi della situazione concorrenziale; allestimento e costi)

- Risorse umane (qualifiche; regolamenti e contratti)

- Direzione e organizzazione (struttura e procedimenti; compiti, responsabilità e competenze)

- Informatica e amministrazione (sistema informatico per lo studio: aspettative e valutazione)

\begin{tabular}{lll}
\hline Data & & \\
\hline K88 & Mercoledì & Chiasso \\
& $\begin{array}{l}\text { 6 giugno 2018 } \\
\text { dalle } 14.00 \text { alle 17.00 }\end{array}$ & FMH Services \\
& (Consulting)
\end{tabular}

Anmeldung/Inscription/Iscrizione www.fmhservices.ch / FMH Consulting Services, 0419250077 